\title{
Designing the Future With the "Delphi Design Sprint": Introducing a Novel Method for Design Science Research
}

\author{
Katja Thoring \\ Anhalt University of Applied Sciences, \\ Delft University of Technology \\ katja@thoring.com
}

\author{
Hermann W. Klöckner \\ Anhalt University of \\ Applied Sciences \\ hwkloeckner@me.com
}

\author{
Roland M. Mueller \\ Berlin School of Economics and Law, \\ University of Twente \\ roland.mueller@hwr-berlin.de
}

\begin{abstract}
This paper introduces a novel innovation method that focuses on the development of future-oriented artifacts. The "Delphi Design Sprint" combines two existing methods-the Delphi method and Design Sprints. The development of the method follows an action research approach and was tested and validated in a university-led design project involving a panel of 20 international experts. This paper introduces the method and describes exemplary results of the project's outcome.
\end{abstract}

\section{Introduction}

The development of new artifacts is one of the major concerns of the Information Systems (IS) field. IS artifacts are defined as constructs, abstracted models, methods, and instantiations [1], and, hence, can include digital products and applications, computer systems, information and communication technologies (ICT), and cyber-physical systems. All these types of artifacts, however, are highly impacted by technological advancements and contextual changes. For example, the 2020 pandemic will most likely have an impact on how we will work and communicate at our future workplaces, but until today, we cannot say exactly, how ICT systems need to adapt to this future scenario. Nonetheless, systematic future studies are less considered in IS curricula and research [2].

Traditional futurology methods, such as trend extrapolation and forecasting, scenario methods, and the Delphi method [3] mainly focus on confirming future scenarios, but not on developing new, future artifacts [4]. Common approaches that do focus on developing future artifacts include speculative and critical design $[5,6]$, and science fiction, which, however, can be regarded as less scientific and are more commonly used in artistic fields.

There exist various methods for testing newly developed artifacts [7], for example through user feedback or usability tests. Such methods are commonly used in the design and IS fields. But when designing for future scenarios, there might not be appropriate users available that would understand the context and the described future problems, and hence, they would not be able to estimate the appropriateness of a proposed design solution. This problem has rarely been discussed in the IS discipline, so far [2].

Hovorka and Peter [8] present an overview of futurology methods and approaches in relation to the IS discipline. In a similar vein, Peter, Riemer, and Hovorka [4] suggest the development of "artifacts from the future" to engage audiences in the discussion of emerging technologies. They present a typology of future artifacts, distinguishing them into historical artifacts, science fiction artifacts, artifacts demonstrating future technology, artifacts creating vicarious experiences, artifacts creating an intended impact, and thought experiments. Such future artifacts could be developed through various approaches like "critical design" [5], "design for debate" [9], or through science fiction [10], but would lack scientific validation. Hence, the authors suggest to expose these "artifacts from the future" to decision-makers, innovators, or policy makers for discussion. We build on this suggestion by introducing a method that involves both - the design of future artifacts, and the discussion and potential validation of the design concepts through expert feedback.

This paper introduces a novel design science-based method that focuses on the development of futureoriented products and services. The "Delphi Design Sprint" combines two existing methods-the Delphi method [3] and Design Sprints [11].

The Delphi method is an approach for future forecasting that involves a panel of experts. Typically, experts rate and comment on given hypotheses or future scenarios in several rounds, with the goal to reach a consensus, which can be discussed in a concluding workshop. 
A Design Sprint is a 5-day workshop that follows a typical design thinking process $[12,13]$ with the goal to develop, prototype, and test an idea.

We combine both approaches into a new method, called Delphi Design Sprint, that consists of future scenarios and developed design concepts, that are presented to and rated by a panel of invited experts. We argue that involving experts from different related fields increases the chances that the developed design solutions would actually become relevant in the future.

As a consequence, the goal of this paper is the introduction and testing of a novel design-science based method for developing artifacts of the future.

\section{Delphi Design Sprint Development}

\subsection{Action Research Methodology}

Our method for developing and testing the Delphi Design Sprint method, is following an action research cycle [14]: (1) Plan, (2) Act, (3) Observe and Evaluate, (4) Reflect and Iterate, and (5) Redesign. Accordingly in Step 1, we developed a Plan for the Delphi Design Sprint method, as described in Section 2.4. In Step 2, Act, we applied the method in an exemplary project in a real-life context. In Step 3, we Observe and Evaluate the developed method and obtained feedback from the participants, both steps outlined in Section 3 of this paper. And in Step 4, we Reflect and Iterate the method, as outlined in the Discussion Section 5. In Step 5, the Plan to Redesign the method is outlined in the "Future Work" subsection. The suggested Delphi Design Sprint method is comprised as a combination of two existing methods, the Delphi method and Design Sprints, which are briefly described in the next subsections.

\subsection{Delphi Method}

The Delphi method is a systematic future forecasting approach mainly relying on a panel of experts. It was developed in the 1950s at the RAND corporation [15]. The name "Delphi" refers to the Oracle of Delphi, a prophecy site in ancient Greece.

The underlying idea of the expert panel is the assumption that a collective opinion would outperform individual expertise [3], a concept nowadays often referred to as "collective intelligence" [16]. Through a structured communication process, often performed via questionnaires, experts discuss a complex topic, develop hypotheses or future scenarios, or rate such hypotheses that are presented to them [3]. The organizing researchers would collect the experts' feedback in an overview. In a subsequent step, the researchers would present this overview back to the experts, so that they could revise their ratings based on the feedback of the other experts. The goal of the process is to reach a consensus among the experts. Hence, a Delphi study is typically constructed around several rounds of expert feedback. The experts usually remain anonymous, sometimes even until after the completion of the study, to avoid bias and to allow for "honest" feedback and for changing one's mind without losing credibility. Therefore, the method reduces the potential negative impact of group dynamics [17]. The advantages of anonymity in design teams is discussed for example in [18]. Moreover, the Delphi method is suitable to involve experts in remote locations as it allows asynchronous responding. According to several sources [19, 20], the Delphi method is most often used to identify problems, determine solutions, or to explore complex, interdisciplinary issues, which involve new or future trends for a given context. However, to the best of our knowledge, the method is rarely used to develop design science-based artifacts.

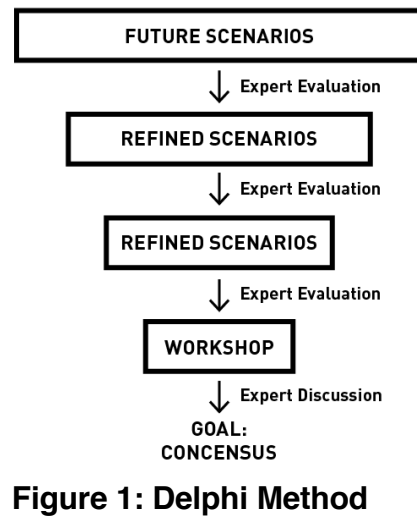

Figure 1 illustrates a typical Delphi study consisting of several rounds and an (optional) concluding workshop.

\subsection{Design Sprints}

Design Sprints are a well-known method for the kick-off of digital product developments, especially in the start-up sector. Developed by Jake Knapp in 2016 at Google Ventures [11], the method focuses on fast iteration and stakeholder involvement and has been the cradle of numerous products, mostly in the digital service design field. The scope of a design sprint is usually defined by focusing on one holistic project kickoff within the frame of one week. The design sprint method is based on the concept that an idea does not need to pass the full product development cycle including "build" and "launch" in order to gain insights for improving the concept.

Each day is usually linked to one specific activity in order to brainstorm, prototype, test and refine a 
project concept. Each activity is dedicated to one day of the five-day workshop: (1) Map, (2) Sketch, (3) Decide, (4) Prototype, and (5) Test. It builds onto the wellknown design thinking process $[12,13]$ and adds the elements of prototyping and testing in order to achieve a high level of confidence in the development and a stronger stakeholder acceptance [21], (Figure 2).

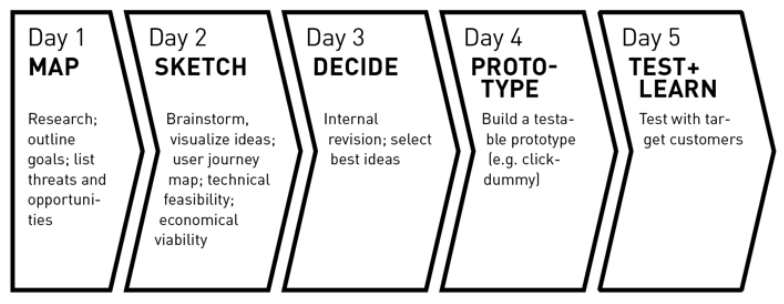

Figure 2: Design Sprint, adapted from [11]

It can be criticized that the design sprint process focusses on speed rather than on scientific rigor and thoroughness. Moreover, the method typically focuses on current contexts, rather than on future scenarios. User feedback in design sprints is typically inquired through direct user testing in day 5. However, we argue that this approach is not adequate for developing future artifacts, because today's users will most certainly not be able to evaluate the usefulness of a solution for contexts they have not yet encountered.

Critique on the design sprint method comes from design practice rather than from academia, though, mainly questioning the lack of scientific rigor and depth [22].

These limitations of both established methods, the Delphi method and design sprints, for developing artifacts for the future, warrant our goal to develop a novel method integrating the best of both approaches - the Delphi Design Sprint.

\subsection{Delphi Design Sprint Method}

We argue that both methods - the Delphi method and design sprints - individually do not provide the adequate toolset to develop future artifacts. To illustrate the theoretical foundation for this identified gap, we refer to the concept of epistemic injustice [23] which describes the phenomenon of inequality of knowledge access. Hermeneutical injustice occurs when people's experiences, needs, and wishes are not well understood, due to historic gaps [23]. We project this concept into the future, where the wishes and needs of future users cannot be researched and understood appropriately, simply because the future is not there yet. For this scenario the - otherwise proven - methods of design thinking and design sprints will not work. The designers of the present cannot empathize or conduct interviews with users of the future. This illustrates the limitations of design sprints when designing for future contexts.

While the Delphi method focuses on confirming future scenarios without creating any artifacts, the design sprint focuses on fast development and iteration of contemporary (non-future) artifacts. A combination of both methods appears to be the sweet spot for developing artifacts for the future.

After a thorough analysis of both existing methods, we identified the core concepts of each and aligned these as intertwined steps within our available timeframe for the intended application of the Delphi Design Sprint. The steps are not simply executed one after the other, but rather conceptualized as iterative loops each influencing the other. We took the Delphi scenarios as an input into the design sprint process to strengthen an expert-validated scope, then validated the concepts in the form of the user journey internally, and finally gathered expert feedback from the visual concept prototypes in order to reiterate the ideas in terms of "desirability" and "feasibility".

We argue, that a Delphi Design Sprint has the potential to merge the academic rigor of the Delphi method with the pace and practical execution of design sprints, fostering a new transfer culture and building a viable iteration bridge between thorough expert research and startup implementation speed. Moreover, the novel method allows the testing of future-targeting design concepts with an expert audience that is able to better estimate the potential applicability of the future concepts than normal users would do.

\section{Application of the Delphi Design Sprint in a Real-Life Project}

In order to validate and test the developed Delphi Design Sprint method, we applied it in a real-life context. This case study represents the "Act" step in the action research cycle. We developed a project in a Master program for design, involving 14 Master students, two researchers (who are both co-authors of this paper), and 20 international experts. Our project's topic was "The Office of the Future" in which design solutions for the office for the upcoming 5 to 15 years should be developed.

In the next subsections, we describe the selection and expertise of the experts, the Delphi Design Sprint cycle, and the project's results.

\subsection{Expert Selection}

Our Delphi Design Sprint involved a panel of 20 international experts, 14 design master students, and 2 researchers. The experts were recruited based on the 
personal network of the two involved researchers as well as through cold-call inquiries. We aimed to build a diverse panel composed of key informants [24] that would represent a broad variety of relevant disciplines with different perspectives. Consequently, among the 20 experts were experts on the methods (e.g. futurologists and design thinking experts), but also experts on the target topic of the project, such as office planners, architects, and technology experts (Figure 3). The invited experts had an average of 19.3 years of professional experience and a median of 15 years. They work in the USA, Germany, and the Netherlands.

The experts were anonymized in the first four rounds to mitigate potential bias and tacit influences between experts. The experts learned about who else participated only in the final workshop.

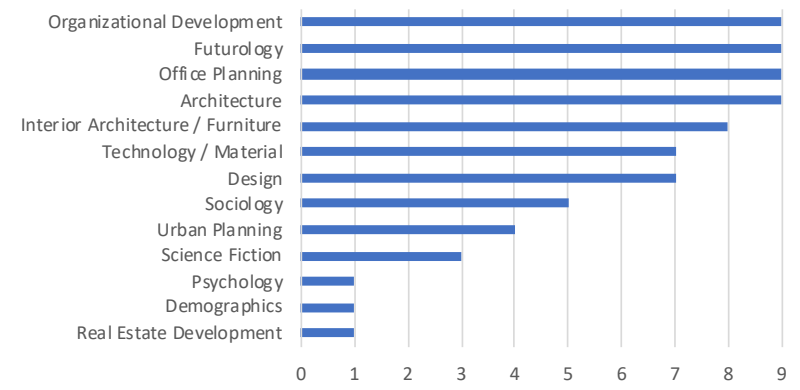

Figure 3. Expertise areas of the 20 involved experts (multiple answers possible)

\subsection{Delphi Design Sprint Project Cycle}

The Delphi Design Sprint process lasted over eight weeks and consisted of a preparation phase, four rounds, and one concluding workshop. In between each round, the student group developed iterations and/or new concepts. Within the design sprints, internal revision rounds winnowed the selection of concepts to be presented to the experts. Figure 4 illustrates the entire process as a flow chart. The left part of the chart illustrates the experts' perspective, and the right part illustrates the internal view.

The response rate for the rounds 1 to 4 was $100 \%$ This extraordinarily high response rate can be attributed to the following facts: The qualitative nature of the study, involving only 20 experts, may have led to a higher engagement of the experts than in a typical (more quantitative) Delphi study, in which the experts were not so closely involved. Furthermore, being part of developing a new method and rating tangible design ideas was seemingly very attractive to the experts. Moreover, the asynchronous schedule of the first four rounds allowed the experts to respond to the questionnaires on their own pace. Finally, we sent up to three follow-up reminders after each round, which was able to engage also those experts who were late with their responses. The response rate for the concluding workshop was lower, at 75\%. Although all 20 experts expressed high interest and willingness to participate, five of them could not clear out the scheduled half day at the suggested date.

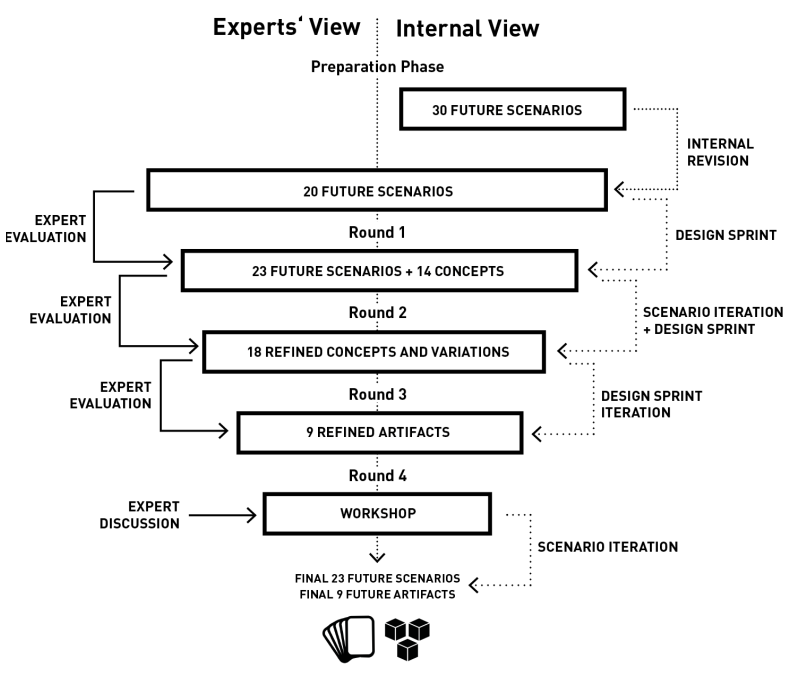

Figure 4. Delphi Design Sprint process flowchart

\subsection{Internal Preparation Phase}

In the internal preparation phase prior to the first round, students developed 30 future scenarios based on literature research and own speculations. In an internal revision with the two involved researchers, these 30 scenarios were narrowed down to 20 to be presented to the experts in round 1 of the Delphi Design Sprint. Our internal exclusion criteria at this point were: (a) concepts that were addressing futures that were too far and not within our 5-15 years time range, and (b) scenarios that were considered by the two researchers as too obvious or already existing.

The developed scenarios were clustered into three categories that also represent different horizons on the future timeline: (1) the post-pandemic workspace (next 1-5 years), (2) workspaces for Generation-Z (next 5-10 years), and (3) the impact of emerging technologies like artificial intelligence or self-driving vehicles (next 1015 years).

\subsection{Development and Iteration of Future Scenarios}

The selected 20 scenarios were presented to the experts as an online questionnaire, each scenario described as approximately 20 to 50 words of text. Each scenario was phrased rather radically, in order to instigate controversial feedback. The experts were asked to rate each scenario based on (a) the likelihood that the scenario would become reality, and (b) how interesting 
they found the scenario in general. Those ratings were presented as a 5-point Likert scale, ranging from "very likely" to "not likely at all", or "very interesting" to "not interesting at all" respectively. Additionally, the experts were invited to provide comments and explanations on their ratings. and to suggest adjustments to the presented scenarios, or additional scenarios. This triangulation of quantitative rating and qualitative feedback resulted in a more complete picture regarding the experts' opinion. An example for a future scenario from the postpandemic category is scenario \#6 "Visible Health":

The physical health of the employees is live-tracked and displayed publicly. People who endanger their colleagues are being radically disciplined. Employees who enter the office sick are laid-off.

This scenario received very negative ratings and, hence, was modified significantly during the subsequent rounds. The final version of the scenario after the concluding workshop was rephrased as follows:

The health of the employees is analyzed discreetly and confidentially by the office's infrastructure. The collected data is pseudonymized and encrypted, and processed and communicated only to the employees themselves. The health of the employees has been sustainably improved through the predictive discrete digital measures.

The experts' comments were (anonymously) presented to all experts in the subsequent rounds to allow for considering new arguments and possibly adjusting one's opinion accordingly. After each round, the results were analyzed and visualized as a graph and presented to all experts. Based on the feedback of the experts, the proposed future scenarios were iterated in order to draw towards a consensus. Three new scenarios were included based on the experts' input.

These iteration steps were processed within Rounds 1 and 2 of the Delphi Design Sprint (as outlined in Figure 4). In the concluding workshop, the scenarios were finalized in a joint discussion with exchange of arguments.

The goal of these steps was to reach a consensus among the experts regarding the future scenarios. Hence, we calculated the standard deviation after each round (Figure 5 shows the standard deviation for the scenarios presented to the experts in round 1).

The average standard deviation for the likelihood of the scenarios improved from Round 1 to Round 2 from 1.31 to 1.21 . In the concluding workshop, the scenarios with the highest standard deviation were discussed among all experts. Through this procedure we were able to find an agreement and we rephrased the scenarios in question accordingly.

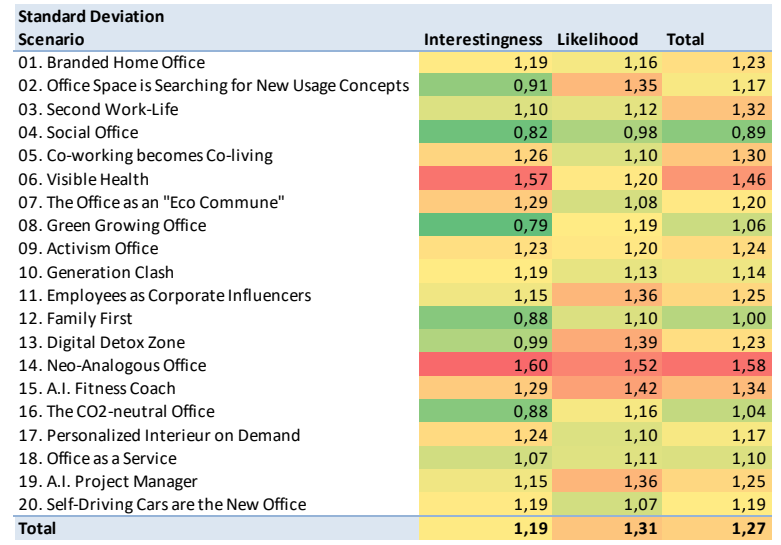

Figure 5: Standard deviation for the likelihood and interestingness of scenarios (round 1)

\subsection{Development and Iteration of the Design Concepts}

Starting from Round 2, students developed design concepts (interventions or solutions) for selected scenarios in one-week design sprints. We call these solutions ideas "concepts" because they were not yet developed into "artifacts" at this stage.

Overall, in three design sprints, the students developed a total of 125 concept sketches (each iteration was counted as a separate concept). These concept sketches passed through several internal revision rounds (that means, not all concepts were presented to the experts). Exclusion criteria at this stage were (a) insufficient clarity of the concept, (b) insufficient visualization quality, or non-existing illustrations, and (c) failure of the concept to convince the majority of the group and teachers.

The design sprint logic applied to the concept development phase helped to identify ideas with a higher potential, specifically through the integration of user journey maps, identifying threats and opportunities, and feasibility/viability discussions (as outlined in Figure 2). Based on these criteria, a majority of the developed concepts was sorted out and not presented to the experts.

Consequently, in Round 2, 14 concept sketches were sent to the experts for feedback; and in Round 3 another 18. Consequently, a total of 32 concept sketches were presented to and rated by the experts.

Each concept was accompanied by an illustration (with a predefined consistent style and format), and a short text of approximately 50 words. A template and design guidelines (dimensions, color scheme, lineweight) were given to the students to ensure comparable visualizations. That way, experts were expected to rate the concepts only, instead of the illustration quality. These design concepts were evaluated by the experts in 
the subsequent Rounds 2 and 3. Figure 6 shows four exemplary concept sketches (out of a total of 32). One concept, the "intelligent desk" (bottom left) will be discussed in more details in Section 4.

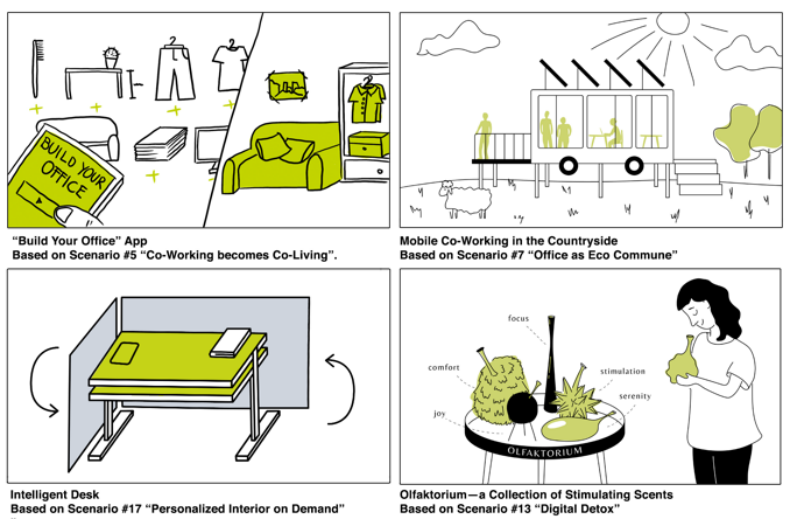

Figure 6. Four exemplary concept sketches

Figure 7 shows the ranking for the concepts presented to the experts in Round 2. It is important to clarify that for the rating of the concepts, our goal was not to reach consensus among the experts. Instead, we were interested in diverging opinions from various perspectives. We assumed that an expert from one field might have different insights on a particular design concept than someone from a different field. Hence, we considered a concept that at least one expert considered as highly relevant and interesting, as still worth developing further.

Consequently, we ranked the design concepts according to three categories: (1) concepts that the majority of experts rated positively, (2) concepts that only a few experts rated positively, and (3) concepts that none of the experts rated positively. Only concepts categorized as (3) were discarded after each round. The concepts from categories (1) and (2) were continued and iterated based on the feedback. The experts were invited to add their comments and feedback on the concepts in the questionnaire so that other experts could reconsider their ratings. Later in the workshop, all experts got the chance to argue for or against certain design concepts and exchange their expertise.

Figure 8 shows the standard deviation of the concept ratings after the third round. It is important to highlight that green here means high agreement but not necessarily a positive rating; and in the same way, red means low agreement but not necessarily negative ratings. For example, Concept 13c ("The Olfaktorium") received a high standard deviation of 1.46 (meaning low agreement). But since there were some experts rating this concept very positively, we kept it in the process to give all experts the possibility to argue for or against this idea.

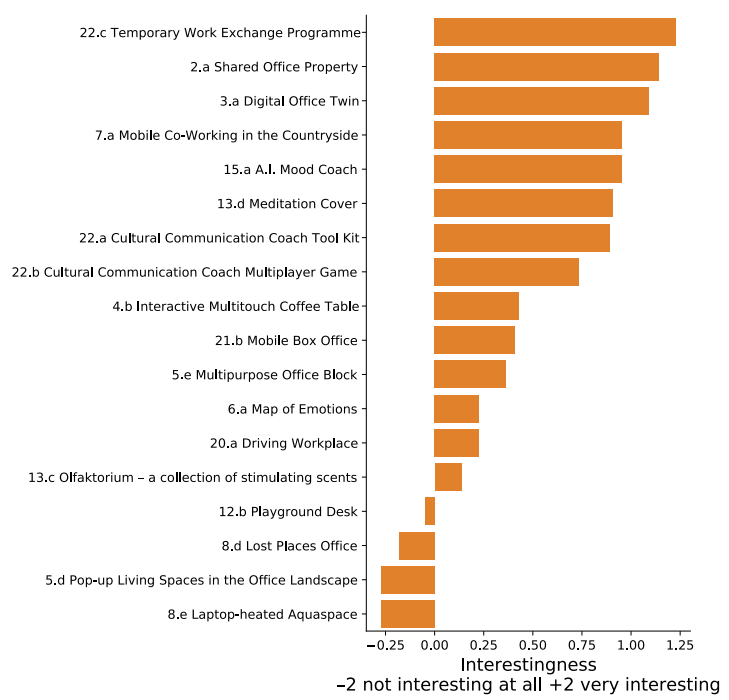

Figure 7. Experts' ranking of concepts (Round 3)

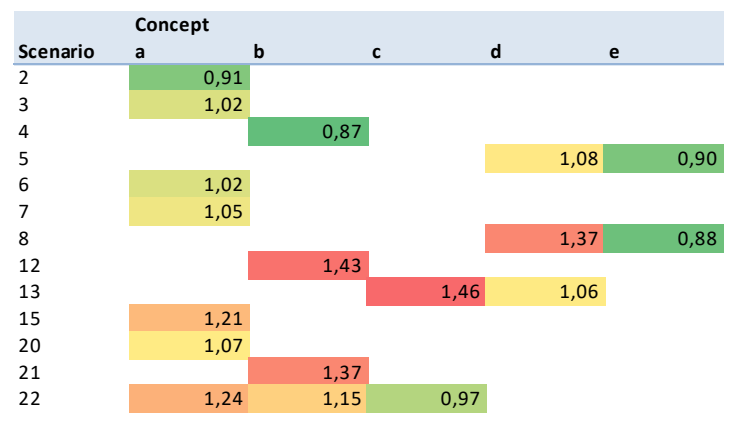

Figure 8. Standard devation of concepts (Round 3)

\subsection{Concluding Workshop}

In the concluding workshop only 15 out of 20 experts were able to attend (75\%). The workshop duration was 4 hours and it was conducted virtually via Zoom and involving a shared Miro board. The workshop was structured as follows: (1) We started with a warmup exercise followed by a self-introduction of all participants. (2) Then we presented a summary of the previous rounds' results, including average ratings and standard deviation for the developed scenarios and design concepts. (3) We then invited a discussion on those scenarios that received a high standard deviation. The experts explained their concerns regarding those scenarios and suggested some adjustments. (4) Then, the students pitched their developed design artifacts. The experts were then asked to vote for their favorite and least favorite design concept and to explain their choices. (5) In a concluding discussion, the experts were invited to discuss and provide answers to three questions: Q1: "My biggest Take-Away from this Workshop is ...", Q2: "The Office of the Future will be ...” and Q3: „For me, the Delphi Design Sprint was ...”. 
Selected responses to these questions will be discussed in the Discussion section of this paper.

\subsection{Results}

As a result of the Delphi Design Sprint, we received feedback by 20 experts not only on the developed future scenarios, but also on the related design concepts (to be developed into artifacts).

Following the workshop, several future scenarios were finally iterated to cater for still ongoing concerns, and then they were illustrated and developed into a card set, consisting of 23 future scenario cards (Figure 9). Each card represents one scenario as a title and illustrative cover; on the reverse side, each scenario is briefly described.

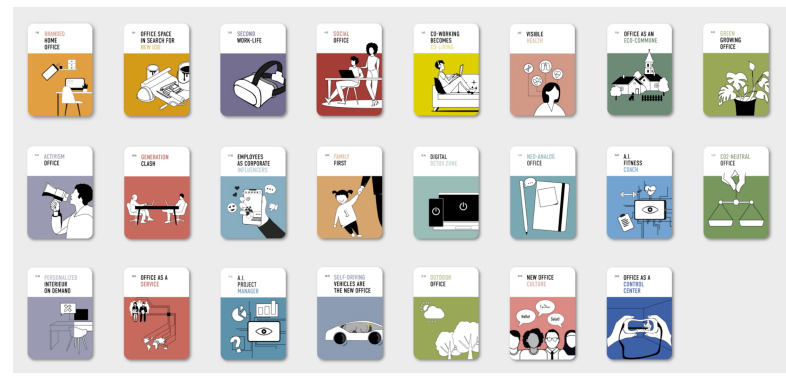

Figure 9. Developed future scenario card set; final scenarios after several iteration rounds

Moreover, nine design artifacts for the office of the future were developed. Figure 10 shows the nine developed concepts as abstracted graphics.

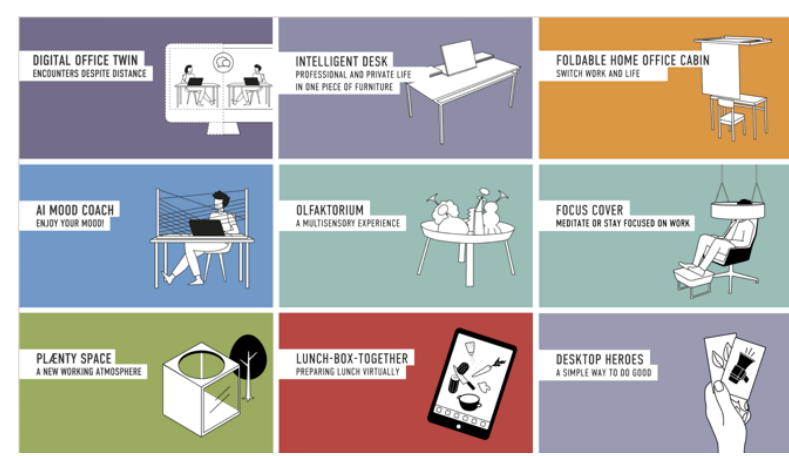

Figure 10. Developed nine future artifacts for the office of the future

Depending on the nature of the concept, the final artifacts were prototyped as physical models, detailed presentation drawings or digital renderings, interactive click-dummies, or atmospheric videos or animations.

In the following section we outline the process of one specific project outcome - the "intelligent desk" by Vanessa Voigt - in order to provide insight into the practical application of the Delphi Design Sprint method.

\section{Exemplary Result "Intelligent Desk"}

The "intelligent desk" is an adaptable hybrid work and leisure workstation. Combining the potentials of origami-inspired folding and in-situ augmented reality, the concept is bridging work and private aspects into one object with numerous use modes for the contemporary home office context. Based on the expert-validated future scenario "Personalized Interior on Demand" (Figure 11), the developed concept contributes to the realization of the scenario. The development process of the concept shall be outlined as follows.

In the first two rounds, the future scenario "Personalized Interior on Demand" has been developed by one student and it passed through two iterations based on the feedback of the expert panel. Both, critical and constructive feedback have been ingested in order to achieve a scenario with both, a high likelihood score and a limited standard deviation among the expert voting. The final scenario description was phrased as follows:

The office of the future, whether at home or in a shared office, will adapt to the spatial needs of employees. The workplace becomes a transforming place. With flexible, hybrid office furnishings, it adapts to the changing requirements of employees.

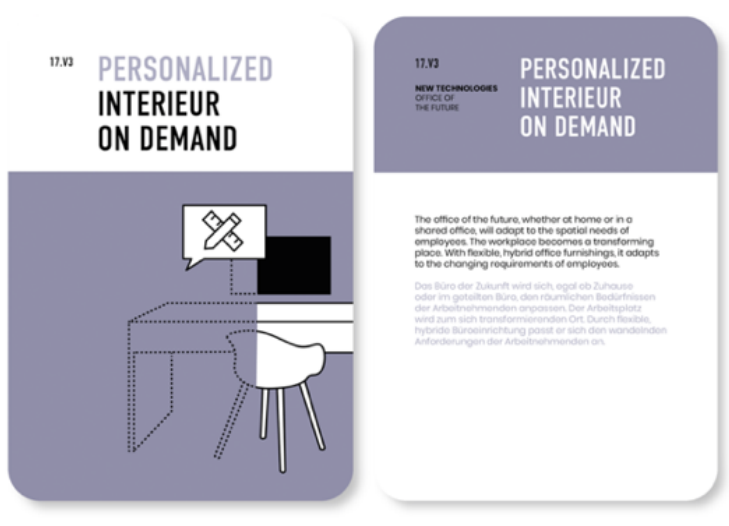

Figure 11. Future scenario \#17: "Personalized interior on Demand"

In the subsequent steps, the scenario was picked up by a different student, reinforcing the interoperable character of the scenarios both within the project and for further work in the field.

The scenario card was used as an input and fed into the first step of the design sprint. This process led to the first concept sketch. Figure 6 (bottom left) shows the initial concept sketch that was presented to the experts along with 13 other concept sketches in Round 2 of the Delphi Design Sprint. The following text accompanied the sketch to explain the idea: 
"Technologies make the desk usable again. There is a pause mode that makes everything disappear and the desk can now be used for activities that are not work-related without any distraction. The user interface switches between different projects according to personalized settings and wishes and only shows what is relevant for the current situation. Notification for other projects can be deactivated in order to avoid a communication overload. For the necessary privacy, there are walls that can be switched on and off at will. It is easier for employees to organize themselves and to switch between breaks or after work."

As the next step of the design sprint, a user journey map (Figure 12), a matrix of threats and opportunities, and different options for technology building blocks have been developed for the internal decision-making step.

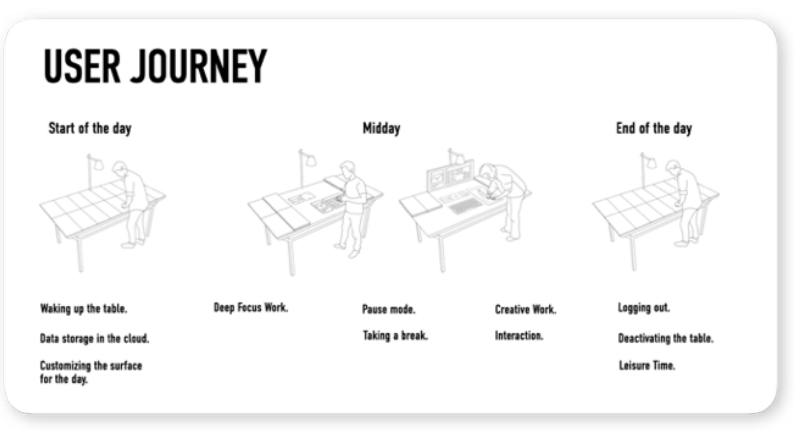

Figure 12. Intelligent desk - user journey map

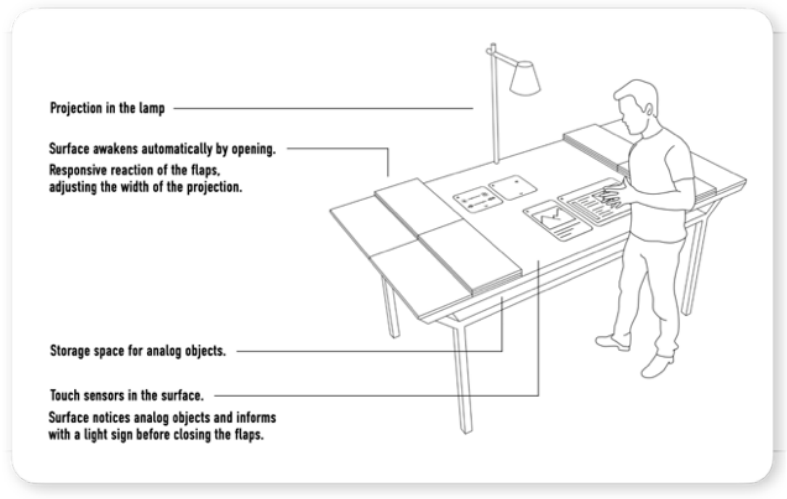

Figure 13. Intelligent desk - concept drawing

Lastly, both a functional prototype and a detailed visual prototype for the testing with the experts as future user representatives were developed (Figures 13 and 14). The experts' feedback to this concept was mainly addressing the adoption of augmented reality (AR) suggesting to increase the likelihood for adoption of the concept not only in the industry but also for potential users.

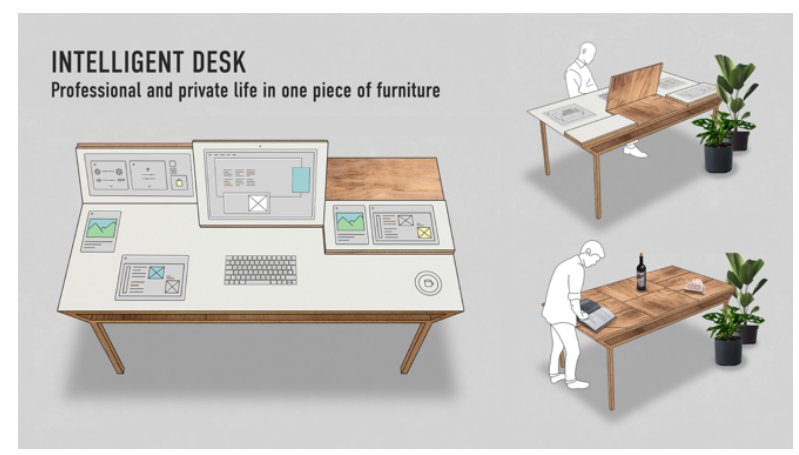

Figure 14. Intelligent desk - prototype visualization

In an agile manner, iterations of the fundamental scenarios have been influxed into the ongoing conceptual process, resulting in an artifact with high acceptance rate (top 4 of all presented concepts) and a low standard deviation (1.04) among experts (Fig. 15).

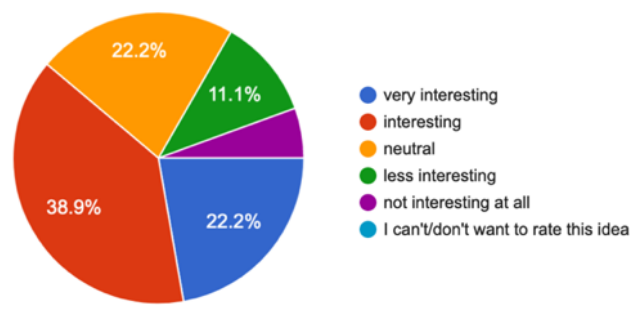

Figure 15. Intelligent desk - ratings by the experts

\section{Reflection and Discussion}

\subsection{Contribution and Relevance}

The developed Delphi Design Sprint presents a combination of best of both worlds: The Delphi study is typically used to validate future scenarios, but does not involve a design science aspect, i.e., the development of new artifacts. The design sprint, however is focusing on development of new artifacts in a fast-paced way, but typically for the presence, when users for user-testing are available and the future is not per se unknown. Hence the suggested Delphi Design Sprint method not only brings more scientific rigor to the design sprint, but it provides also a method to develop future products for future scenarios, for which proficient users to test and validate the developed solutions are typically not yet available.

We argue that the developed method contributes to research and practice in several ways: First, the method can be adapted and transferred to different contexts in order to develop future-focused artifacts (see Section 5.4 "Actionable Advice"). For start-up companies who would normally focus on a design sprint to kick-off their product development process and to create a testable prototype in a very fast-paced manner, the Delphi Design Sprint has several advantages: (1) The focus on 
future scenarios would give them a competitive advantage compared to established companies or to other start-ups who might not focus on visionary future scenarios. (2) The involvement of experts and the resulting expert-validation of their concepts would provide them with a profound basis to approach venture capitalists and other funding opportunities.

Secondly, the 23 expert-validated future scenario cards from this project can be of use for other researchers and designers who want to develop artifacts for the future workplace, a very timely and relevant topic, especially for the upcoming post-pandemic times.

Thirdly, the method can help innovators and companies when developing artifacts at the fuzzy front end of innovation [25]. The method can facilitate the development of a large number of ideas for rather unknown and "fuzzy" contexts.

Lastly, the involvement of rigorous expert feedback may help overcome design fixation [26], because they might provide more unbiased and "honest" feedback than internal revisions would typically provide.

\subsection{Experts' Feedback on the Method}

In the concluding workshop the experts provided us with feedback on the method itself.

Most experts mentioned that they enjoyed the multidisciplinary approach of the method and that they were excited about being involved into a design project. They also mentioned concerns regarding the required time-involvement ("at least 1 hour per round"), which was for many of them difficult to accomplish. Several experts expressed the wish to meet the other experts earlier in the process. One suggestion was to kick-off the project with a workshop in which the experts could provide their expertise as an input for the scenario development. Finally, some experts criticized the questionnaire's formulation of "likelihood" and "interestingness" of scenarios, which they found difficult to distinguish. These comments and suggestions for improving the method shall be considered for future iterations of the method. Overall, the Delphi Design Sprint method was described as enriching and inspiring by most experts, and considered as a new means to "bring reality to imagination."

\subsection{Future Work}

As suggested by the experts' feedback, we would consider to involve the experts into the scenariodevelopment process. Rather than developing our own scenarios, we would invite experts to deliver input on their expertise and develop the scenarios together with the researchers and designers. In order to keep the benefit of anonymity in the beginning (as outlined in
Section 2), we recommend this initial input to be delivered through questionnaires rather than a workshop.

Secondly, the phrasing of the questionnaire needs to be carefully reconsidered. In an iterated version of the method, we would limit to only one rating scale (likelihood of the future scenario) and not ask for how interesting the expert would find the scenario.

Thirdly, as a next step we suggest to conduct user testing with the developed prototypes. Since normal users might not be able to adequately evaluate the potential of the future concepts (as outlined in Section 2.4), we suggest to involve lead-users [27] who can be considered as "early adopters" of future trends.

Finally, as the next step for the Delphi Design Sprint, we envision the development of a (digital or analog) tool to facilitate the process.

\subsection{Actionable Advice}

Based on our own reflection and the experts' feedback on the method, we suggest the following steps and criteria for conducting a Delphi Design Sprint.

Steps: (1) Recruit a panel of experts with diverse perspectives on the topic. (2) Develop future scenarios based on the experts' input, via online questionnaires (one round). (3) Validate scenarios through experts' feedback via online questionnaires (one or more rounds). (4) Develop design concepts in design sprints (one or more internal rounds). (5) Validate the design concepts through experts' feedback via online questionnaires (one or more rounds). (6) Conduct a concluding workshop for final discussion.

Criteria: (a) Compose a multidisciplinary expert panel to invite different perspectives. (b) Keep experts anonymous until the concluding workshop to mitigate bias. (c) Allow for asynchronous participation (except for the workshop) to cater different time schedules. (d) Invite mixed method feedback (quantitative and qualitative) for triangulation. (e) Keep a consistent format and style for all presented concepts to ensure comparability.

\subsection{Limitations}

We are aware that a method is only as good as the quality of the resulting outcomes. However, it lies in the nature of future forecasting that one cannot evaluate the results in conclusion, before the future has actually occurred. Hence, we cannot finally evaluate the quality of the developed artifacts and how the method may have helped to shape them. Time will tell if we actually see some of the developed design concepts successfully implemented in the near future. 
A second limitation of our study is the fact that we conducted the Delphi Design Sprint in an educational context with Master students, rather than in a professional context. However, we argue that through the involvement of 20 renowned international experts, we were able to compensate for this potential deficit. Moreover, the Master students each had already a professional degree and some work experience.

Finally, we conducted only one practical application of the method. Future work will need to include further test rounds to validate and iterate the method further.

\section{Conclusions}

In this paper, we introduce a novel design science research method, called the Delphi Design Sprint, that aims at developing artifacts for the future. The method was developed by passing through an action research cycle, applying and testing it in a real-life student project involving 20 renowned experts.

The development of future artifacts is an important goal for the IS discipline. Technologies are developing fast, and also contexts are changing rapidly (we know this specifically since the outbreak of the current pandemic). Designing for future scenarios in a fastpaced manner and with expert-feedback can lead to a competitive advantage for startups and established companies alike. The developed Delphi Design Sprint method facilitates this fast-paced, yet rigorous development of artifacts for unknown future scenarios.

\section{References}

[1] A. Hevner, S. T. March, J. Park, and S. Ram, "Design science in information systems research," MIS Quarterly, vol. 28, no. 1, pp. 75-105, 2004.

[2] E. Carmel, M. Avital, P. Gray, J. Kallinikos, and J. L. King, "Teaching Foresight and the Future," in Researching the Future in Information Systems, Berlin, Heidelberg, 2011, pp 291-293.

[3] H. A. Linstone and M. Turoff, The delphi method. AddisonWesley Reading, MA, 1975.

[4] S. Peter, K. Riemer, and D. Hovorka, "Artefacts from the Future: Engaging Audiences in possible Futures with Emerging Technologies for better Outcomes," ECIS Proceedings, Jun. 2020.

[5] L. Jakobsone, "Critical design as approach to next thinking," The Design Journal, vol. 20, no. sup1, pp. S4253-S4262, Jul. 2017.

[6] A. Dunne and F. Raby, Speculative everything: design, fiction, and social dreaming. MIT press, 2013.

[7] J. Venable, J. Pries-Heje, and R. Baskerville, "FEDS: a Framework for Evaluation in Design Science Research," European Journal of Information Systems, vol. 25, no. 1, pp. 77-89, Jan. 2016.
[8] D. Hovorka and S. Peter, "How the Future is Done," Proceedings of the 52nd Hawaii International Conference on System Sciences (HICSS), Jan. 2019.

[9] A. Dunne, "Design for debate," Architectural Design, vol. 78 , no. 6 , pp. 90-93, 2008

[10] K. Steinmüller, "Science Fiction and Science in the Twentieth Century," in Companion to Science in the Twentieth Century, Taylor \& Francis, 2003.

[11] J. Knapp, J. Zeratsky, and B. Kowitz, Sprint: How to Solve Big Problems and Test New Ideas in Just Five Days, Export. New York: Simon \& Schuster, 2016.

[12] T. Brown, "Design Thinking," Harvard Business Review, vol. 86, no. 6, pp. 84-92, 2008.

[13] K. Thoring and R. M. Müller, "Understanding Design Thinking: A Process Model based on Method Engineering," Proceedings of the 13th International Conference on Engineering and Product Design Education (E\&PDE), London, UK, 2011.

[14] S. Kemmis, R. McTaggart, and R. Nixon, The Action Research Planner: Doing Critical Participatory Action Research. Singapore: Springer, 2013.

[15] N. Dalkey and O. Helmer, "An Experimental Application of the DELPHI Method to the Use of Experts," Management Science, vol. 9, no. 3, pp. 458-467, 1963.

[16] J. M. Leimeister, "Collective intelligence," Business \& Information Systems Engineering, vol. 2, no. 4, pp. 245-248, 2010.

[17] D. Fink-Hafner, T. Dagen, M. Doušak, M. Novak, and M. Hafner-Fink, "Delphi Method: Strengths and Weaknesses," Advances in Methodology \& Statistics / Metodoloski zvezki, vol. 16, no. 2, pp. 1-19, 2019.

[18] A. L. Aguerrevere, J. Alleblas, R. M. Mueller, M. Graves, and K. Thoring, "The Impact of Status Anonymity on Team Dynamics in Co-Creation Workshops," in Proceedings of the Design Society: DESIGN Conference, May 2020, vol. 1, pp. $1465-1474$

[19] L.-H. Chang and G. Gable, "A critique of the Delphi method in the context of IS key issues studies," PACIS 2000 Proceedings, p. 82, 2000.

[20] W. Scholl, C. König, B. Meyer, and P. Heisig, "The future of knowledge management: an international delphi study," Journal of knowledge management, 2004.

[21] C. M. M. de Sá Araújo, I. M. Santos, E. D. Canedo, and A. P. F. de Araújo, "Design Thinking Versus Design Sprint: A Comparative Study," in International Conference on Human-Computer Interaction, 2019, pp. 291-306.

[22] A. Abut, "Design Sprints: Pros, Cons \& Alternatives." https://alabut.com/writing/designsprint (accessed Jun. 13, 2021).

[23] M. Fricker, Epistemic Injustice: Power and the Ethics of Knowing. Oxford: Oxford University Press, 2009.

[24] R. K. Yin, Case Study Research, 4th ed. Newbury Park: Sage, 2009.

[25] P. A. Koen et al., "Fuzzy Front End: Effective Methods, Tools, and Techniques," in The PDMA ToolBook for New Product Development, P. Belliveau, A. Griffin, and S. Somermeyer, Eds. New York: John Wiley \& Sons, 2002, p. 32.

[26] A. T. Purcell and J. S. Gero, "Design and other types of fixation," Design Studies, vol. 17, no. 4, pp. 363-383, 1996.

[27] G. L. Urban and E. von Hippel, "Lead User Analyses for the Development of New Industrial Products," Management Science, vol. 34, no. 5, pp. 569-582, May 1988, doi: 10.1287/mnsc.34.5.569. 